\title{
Performance Limits on Beamforming with Finite Rate Feedback for Multiple Antenna Systems
}

\author{
K. Kiran Mukkavilli, A. Sabharwal and B. Aazhang \\ Rice University \\ Houston, TX 77005 \\ \{mkkiran, ashu, aaz\}@rice.edu
}

\author{
E. Erkip \\ Polytechnic University \\ Brooklyn, NY 11201 \\ elza@poly.edu
}

\begin{abstract}
In this work, we address the problem of evaluating the outage performance of beamformers designed for multiple transmit and single receive antenna systems when quantized channel state information is available at the transmitter. We present a tight universal lower bound for the outage probability of an arbitrary beamformer codebook consisting of a finite number of beamforming vectors. We also characterize the loss in outage performance due to the quantized nature of the channel information available at the transmitter. In particular, we show that the outage probability of beamforming based on quantized channel information approaches the performance of perfect information beamforming as $(t-1) 2^{-\frac{B}{t-1}}$, where $B$ is the number of bits used to quantize the vector channel and $t$ is the number of transmit antennas.
\end{abstract}

\section{Introduction}

Recently, space time coding schemes for multiple antenna systems have received wide attention following the works of Telatar [1] and Tarokh et al. [2]. In the analysis of space time codes, it is assumed that the channel state information at the receiver (CSIR) is perfect while the channel state information at the transmitter (CSIT) is limited to channel statistics only (actual channel realization is unknown to the transmitter). CSIR requires training symbols for channel estimation while CSIT requires resources in the form of feedback from the receiver to the transmitter. It has been observed that significant performance gains, at much lower complexity at times, can be achieved in the presence of CSIT. Telatar [1] analyzed perfect CSIT while Narula et al. [3] analyzed several schemes with imperfect CSIT, with multiple transmit and a single receive antenna. Power control algorithms, which minimize probability of outage or maximize mutual information, were designed in $[4,5]$. The performance of beamforming with different kinds of structure on feedback information, like channel phases or channel amplitude, was analyzed in $[6,7]$. The conditions for the optimality of beamforming, with mutual information as the performance metric, was studied in [8-10].

In practice, the capacity of the feedback channel is limited to a few bits and hence it is important to analyze the performance of transmission schemes with constraints on the feedback channel capacity. In this work we assume perfect channel information at the receiver. We further assume that quantized channel information is available at the transmitter via an errorfree feedback channel of capacity $B$ bits/frame. This feedback model is appropriate for a frequency division duplex (FDD) system where the channels for the uplink and the downlink are different, so that training in the reverse direction is not useful.

In this work, we present tight lower bounds on the outage performance of beamforming schemes in the presence of quantized feedback. We also quantify the performance gains with feedback as a function of the number of feedback bits and the number of transmit antennas, when beamforming schemes are used for transmission. The proposed analysis is useful in determining the extent of feedback information required to achieve a certain frame error performance with a given number of transmit antennas. Actual construction of near-optimal beamformer codebooks with finite number of beamforming vectors can be found in [11].

The paper is organized as follows. The system model is given in Section 2. In Section 3, we derive a lower bound on outage performance and quantify the gains of finite size beamformer codebooks. We conclude in Section 4.

\section{System Model}

Consider a wireless communication system with $t$ transmit antennas and a single receive antenna. We assume a narrowband channel so that the channel is frequency non-selective. Let $\mathbf{h}=\left[h_{1}, h_{2}, . . h_{t}\right]$ be the $1 \times t$ channel vector from the transmitter to the receiver, where each $h_{i}$ is a circularly symmetric com- 
plex Gaussian random variable with zero mean and unit variance per complex dimension. We assume that the channel is statistically independent across the antennas, i.e., $h_{i}$ and $h_{j}$ are independent for $i \neq j$. Let $X=\left[x_{1}, x_{2}, \ldots x_{t}\right]$ be the $1 \times t$ transmitted vector, where $x_{i}$ denotes the transmission from the $i$ th antenna. The average transmission power is constrained to $P$ so that

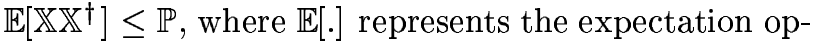
eration while the superscript $\dagger$ represents conjugate transposition of a matrix. When $X$ is transmitted, the corresponding received signal $y$ is given by

$$
y=\mathbf{h} X^{\dagger}+\eta,
$$

where $\eta$ is the additive noise which is assumed to be circularly symmetric complex Gaussian random variable with zero mean and unit variance. Further, the channel statistics are assumed to be quasi-static in time, i.e., the channel realization is independent across different frames while the channel does not vary significantly within a given frame. It is assumed that perfect channel state information is available at the receiver. A feedback channel with a capacity of $B$ bits/frame is available in the system which conveys quantized channel state information to the transmitter. Hence, the channel information at the transmitter comprises of the channel statistics and $B$ bits of information about the channel realization made available by the feedback channel.

The transmission strategy is fixed to be beamforming. Corresponding to the $B$ bits of feedback, we have a beamformer codebook $\mathcal{C}$ comprising of $N=2^{B}$ beamforming vectors. The elements in the codebook $\mathcal{C}$ are given by $\left\{C_{1}, C_{2}, \ldots C_{N}\right\}$, where $C_{i} \in \mathbb{C}^{t}$, for each $i$. With beamforming strategy, the transmission vector $X$ in (1) can be written as $X=C_{i} x$, where $C_{i}$ is the beamforming vector and $x$ is the information bearing scalar. Note that the transmitter chooses the appropriate beamforming vector $C_{i}$ from the beamformer codebook $\mathcal{C}$ based on the $B$ feedback bits. Finally, our performance metric is outage probability $[1,12]$, where outage is defined as the event of channel not being able to support the desired rate of transmission $R$.

\section{Performance limits on beamforming}

In this section, we characterize the outage performance of any finite size beamformer codebook with a lower bound. The lower bound allows us to investigate the performance of finite size beamformer codebooks as a function of the number of feedback bits as well as the number of transmit antennas. In addition, the proof methodology of the lower bound also leads to a design criterion for near-optimal beamformer codebooks [11].

\subsection{Quantizer design}

Let $\mathcal{C}=\left\{C_{1}, C_{2}, \ldots C_{N}\right\}$ be an arbitrary beamformer as described in Section 2. Without loss of generality, we assume that the quantizer codebook at the receiver to be the same as the beamformer codebook at the transmitter. Hence, the feedback information of $B$ bits will be used to describe the label $i$ of the beamforming vector $C_{i}$. Let $Q: \mathbb{C}^{t} \rightarrow \mathcal{C}$ be any quantizer. Then the received signal for a given channel realization $\mathbf{h}$ can be written as

$$
y=\langle\mathbf{h}, Q(\mathbf{h})\rangle x^{*}+\eta,
$$

where $\langle u, v\rangle=u v^{\dagger}$. Note that (2) is a Gaussian channel for a given channel realization $\mathbf{h}$ and a fixed quantizer $Q$ so that mutual information conditioned on $\mathbf{h}$ and $Q$ is maximized by choosing Gaussian distribution for $x$. In particular, we have

$$
\max _{P(X \mid \mathbf{h}, Q)} I(X ; y \mid \mathbf{h}, Q)=\log _{2}\left(1+P|\langle\mathbf{h}, Q(\mathbf{h})\rangle|^{2}\right) .
$$

It can be shown that a good quantizer $Q^{*}$ which minimizes the outage probability is given by the inner product norm. The structure of the quantizer $Q^{*}$ is given by the following lemma.

Lemma 1 (Optimal Quantizer [11])

For any beamformer codebook $\mathcal{C}=\left\{C_{1}, C_{2}, \ldots C_{N}\right\}$, the outage probability is minimized by choosing for each channel realization $\mathbf{h}$, the vector $C_{i} \in \mathcal{C}$ which maximizes $\left|\left\langle\mathbf{h}, C_{i}\right\rangle\right|$.

The Voronoi region corresponding to a vector $C_{i}$ and a channel norm $\|\mathbf{h}\|_{2}=\sqrt{\gamma}$ with the quantizer $Q^{*}$, denoted by $V_{i}(\gamma)$, is given by

$$
V_{i}(\gamma)=\left\{\mathbf{h}:\|\mathbf{h}\|^{2}=\gamma,\left|\left\langle\mathbf{h}, C_{i}\right\rangle\right| \geq\left|\left\langle\mathbf{h}, C_{j}\right\rangle\right|, j \neq i\right\} .
$$

Note that the for a given $\gamma$, the region $V_{i}(\gamma)$ is a portion of the surface of the hypersphere given by $\|\mathbf{h}\|^{2}=\gamma$. Also, let $A\left(V_{i}(\gamma)\right)$ denote the surface area of $V_{i}(\gamma)$.

\subsection{Outage regions}

We will now characterize the subsets of $V_{i}(\gamma)$ which can support a desired rate of transmission $R$, referred to as no-outage region and denoted by $\Theta_{i}(\gamma)$. The complement of the no-outage region is the outage region, region which cannot support the rate of transmission $R$ and hence results in outage. The characterization of the no-outage regions turns out to be much 
simpler than the outage regions and hence we take that approach.

It is clear from (3) that outage does not occur when $C_{i}$ is chosen for transmission if and only if $\left|\left\langle\mathbf{h}, C_{i}\right\rangle\right|^{2} \geq$ $\frac{2^{R}-1}{P}$ so that we have

$$
\Theta_{i}(\gamma)=\left\{\mathbf{h}: \mathbf{h} \in V_{i}(\gamma),\left|\left\langle\mathbf{h}, C_{i}\right\rangle\right|^{2} \geq \gamma_{0}\right\}
$$

where $\gamma_{0}=\frac{2^{R}-1}{P}$.

Let $P_{\text {out }}^{c}\left(R, P \mid C_{i},\|\mathbf{h}\|^{2}=\gamma\right)$ denote the probability that outage does not take place when $C_{i}$ is chosen for transmission and the channel norm is $\sqrt{\gamma}$. Then, we have,

$$
\begin{aligned}
P_{\text {out }}^{c}( & \left.R, P \mid C_{i},\|\mathbf{h}\|^{2}=\gamma\right)= \\
& \operatorname{Prob}\left[\mathbf{h} \in \Theta_{i}(\gamma) \mid \mathbf{h} \in V_{i}(\gamma),\|\mathbf{h}\|^{2}=\gamma\right] \\
= & \frac{\operatorname{Prob}\left[\mathbf{h} \in \Theta_{i}(\gamma), \mathbf{h} \in V_{i}(\gamma) \mid\|\mathbf{h}\|^{2}=\gamma\right]}{\operatorname{Prob}\left[\mathbf{h} \in V_{i}(\gamma) \mid\|\mathbf{h}\|^{2}=\gamma\right]} \\
= & \frac{\operatorname{Prob}\left[\mathbf{h} \in \Theta_{i}(\gamma) \mid\|\mathbf{h}\|^{2}=\gamma\right]}{\operatorname{Prob}\left[\mathbf{h} \in V_{i}(\gamma) \mid\|\mathbf{h}\|^{2}=\gamma\right]}, \text { since } \Theta_{i}(\gamma) \subseteq V_{i}(\gamma) \\
= & \frac{A\left(\Theta_{i}(\gamma)\right)}{A\left(V_{i}(\gamma)\right)} .
\end{aligned}
$$

At this point, it is worthwhile to note that the distribution of $\mathbf{h}$ on the surface of the hypersphere $\|\mathbf{h}\|^{2}=\gamma$ is uniform so that the probability of choosing $C_{i}$ for transmission is given by

$$
P\left(C_{i}\|\| \mathbf{h} \|^{2}=\gamma\right)=\frac{A\left(V_{i}(\gamma)\right)}{A(\gamma)},
$$

where $A(\gamma)$ is the surface area of the $t$-dimensional complex hypersphere of radius $\sqrt{\gamma}$. Finally, the probability of no-outage resulting from the beamformer codebook $\mathcal{C}$ conditioned on $\gamma$ is given by the total probability rule as

$$
\begin{aligned}
& P_{\text {out }}^{c}\left(R, P \mid\|\mathbf{h}\|^{2}=\gamma\right) \\
& \left.\left.=\sum_{i=1}^{N} P\left(C_{i} \mid\|\mathbf{h}\|^{2}=\gamma\right)\right) P_{\text {out }}^{c}\left(R, P \mid C_{i},\|\mathbf{h}\|^{2}=\gamma\right)\right) \\
& =\sum_{i=1}^{N} \frac{A\left(V_{i}(\gamma)\right)}{A(\gamma)} \frac{A\left(\Theta_{i}(\gamma)\right)}{A\left(V_{i}(\gamma)\right)} \\
& =\frac{\sum_{i=1}^{N} A\left(\Theta_{i}(\gamma)\right)}{A(\gamma)} .
\end{aligned}
$$

\subsection{Outage probability with feedback}

It can be shown [11] that the right hand side of (7) can be bounded in different intervals of $\gamma$ leading to the following set of bounds.

$$
P_{\text {out }}^{c}\left(R, P \mid\|\mathbf{h}\|^{2}=\gamma\right)
$$

$$
\begin{aligned}
& =0, \gamma<\gamma_{0} \\
& \leq N\left(1-\frac{\gamma_{0}}{\gamma}\right)^{(t-1)}, \gamma_{0}<\gamma<\gamma_{1} \\
& \leq 1, \gamma_{1}<\gamma
\end{aligned}
$$

where $\gamma_{1}=\frac{\gamma_{0}}{\left(1-\left(\frac{1}{N}\right)^{\left(\frac{1}{t-1}\right)}\right)}$. We can now integrate (8) over the distribution of $\gamma$ (note that $\gamma$ is chi-squared with $2 t$ degrees of freedom) to arrive at the following theorem.

\section{Theorem 1 (Outage lower bound)}

Consider a wireless system with $t$ transmit antennas along with a single receive antenna transmitting at rate $R$ bits/sec/Hz and SNR P over a quasi-static Rayleigh fading channel. The outage probability of such a system employing a beamformer codebook with $N$ beamforming vectors (corresponding to $\log _{2}(N)$ bits of feedback per frame) is bounded below as

$$
\begin{aligned}
& P_{\text {out }}(R, P) \geq \\
& \quad 1-N e^{-\gamma_{0}}+e^{-\gamma_{1}} \sum_{k=0}^{t-1}\left(\frac{N\left(\gamma_{1}-\gamma_{0}\right)^{k}-\gamma_{1}^{k}}{k !}\right)(9)
\end{aligned}
$$

where

$$
\gamma_{0}=\frac{2^{R}-1}{P} \text { and } \gamma_{1}=\frac{\gamma_{0}}{\left(1-\left(\frac{1}{N}\right)^{\left(\frac{1}{t-1}\right)}\right)}
$$

Proof See Appendix.

Note that the lower bound is true for an arbitrary beamformer codebook consisting of $N$ beamforming vectors for all regimes of SNR and the number of transmit antennas $t$. We will now evaluate the outage probability of beamforming when perfect channel information is available at the transmitter. Note that the perfect channel information can be treated as the case when $N$ is made very large and hence serves as a useful reference for the finite feedback case. It can be shown easily [11] that the probability of outage in this case is given by

$$
\begin{aligned}
P_{\text {out }}^{\infty}(R, P) & =\operatorname{Prob}\left[\log _{2}\left(1+P\|\mathbf{h}\|^{2}\right)<R\right] \\
& =\operatorname{Prob}\left[\gamma<\frac{2^{R}-1}{P}\right] \\
& =1-e^{-\gamma_{0}} \sum_{k=0}^{t-1} \frac{\gamma_{0}^{k}}{k !}
\end{aligned}
$$

Figure 1 compares the lower bound on the performance of beamforming with finite rate feedback, given by Theorem 1, with the case of perfect channel information given in (11). In Figure 1, the number 
of transmit antennas is four while the number of receive antennas is one and the SNR is $10 \mathrm{~dB}$ with $R=2 \mathrm{bits} / \mathrm{sec} / \mathrm{Hz}$. We see that the performance of the beamforming with finite rate feedback comes very close to that of beamforming with perfect channel information for about 7 to 8 bits of feedback information. Further, the marginal gain obtained by the addition of more feedback bits is small.

\subsection{High SNR approximation}

Further insights into the performance of the finite size beamforming codebooks can be obtained by analyzing (9) and (11) under high SNR conditions. It can be shown that (9) approximates the outage performance of good beamformers for sufficiently large $N$ so that we have

$$
\begin{aligned}
P_{\text {out }}(R, P) \approx & 1-N e^{-\gamma_{0}} \\
& +e^{-\gamma_{1}} \sum_{k=0}^{t-1}\left(\frac{N\left(\gamma_{1}-\gamma_{0}\right)^{k}-\gamma_{1}^{k}}{k !}\right) \\
= & \sum_{k=t}^{\infty}\left(\frac{\gamma_{1}^{k}-N\left(\gamma_{1}-\gamma_{0}\right)^{k}}{k !}\right),
\end{aligned}
$$

where we have used the series expansion of $e^{x}$ to arrive at (12). Note that $\gamma_{0}=\frac{2^{R}-1}{P}$ so that as $P$ increases, $\gamma_{0}$ goes to zero. Hence, ignoring the higher powers of $\gamma_{0}$, we have

$$
\begin{aligned}
P_{\text {out }}(R, P) & \approx \frac{\gamma_{0}^{t}}{t !} \frac{1-N\left(\frac{1}{N}\right)^{\frac{t}{t-1}}}{\left(1-\left(\frac{1}{N}\right)^{\frac{1}{t-1}}\right)^{t}} \\
& =\frac{\gamma_{0}^{t}}{t !} \frac{1}{\left(1-\left(\frac{1}{N}\right)^{\frac{1}{t-1}}\right)^{t-1}}
\end{aligned}
$$

A similar analysis of the outage probability of beamforming with perfect feedback results in

$$
P_{\text {out }}^{\infty} \approx \frac{\gamma_{0}^{t}}{t !} \text {. }
$$

It follows from (13) and (14) that the loss in outage performance at high SNR due to finite nature of the feedback is captured by

$$
L(t, N)=\left(1-\left(\frac{1}{N}\right)^{\frac{1}{t-1}}\right)^{-(t-1)} .
$$

Note that $L(t, N) \geq 1$ and decays to 1 as $N$ increases for a fixed $t$. We can now define a distortion measure $F(B)$ for channel quantization based on the outage performance of the best resulting beamformer. In particular, for large SNR we define,

$$
F(B) \triangleq \frac{P_{o u t}^{B}(R, P)-P_{o u t}^{\infty}(R, P)}{P_{o u t}^{\infty}(R, P)},
$$

where $P_{\text {out }}^{B}(R, P)$ is the outage probability of a beamformer codebook with $N=2^{B}$ vectors. Using (13) and (14) along with the definition of $F(B)$, it can be easily shown that, for sufficiently large $B$, we have

$$
F(B) \approx(t-1) 2^{-\frac{B}{t-1}} .
$$

Hence, the relative performance loss of quantized beamforming compared to perfect channel feedback decays exponentially with the number of feedback bits $B$, scaled by $(t-1)$, for sufficiently large $B$ and SNR. Further discussions on the distortion metric and comparisons with the classical rate distortion function (defined for mean squared error) can be found in [11].

\section{Conclusions}

We have presented a universal lower bound for the outage performance of an arbitrary beamformer with finite number of beamforming vectors. The bound is fairly tight in all regimes of SNR and the number of beamforming vectors as well as different number of transmit antennas. We have also shown that the outage performance of beamforming based on finite rate feedback channel approaches the outage performance of beamforming based on perfect channel information exponentially in the number of feedback bits scaled by the number of transmit antennas. The proof methodology for the lower bound also explains the structure of near-optimal beamformer codebooks. Further results on the design criterion and the construction of the beamformer codebooks with a finite rate feedback channel can be found in [11]. Several interesting problems such as the joint design of beamformers and power control algorithms for single as well as multiple receive antennas are currently under investigation.

\section{A Proof of Theorem 1}

Let $\mathcal{C}$ be an arbitrary beamformer codebook with $N$ beamforming vectors. Let $P_{\text {out }}^{c}(R, P)$ be the probability of no-outage when $\mathcal{C}$ is used for beamforming. We have

$$
P_{\text {out }}^{c}(R, P)=\int_{\gamma=0}^{\infty} P_{\text {out }}^{c}\left(R, P \mid\|\mathbf{h}\|^{2}=\gamma\right) p_{\gamma}(\gamma) d \gamma
$$

Using the upper bound on $P_{\text {out }}^{c}\left(R, P \mid\|\mathbf{h}\|^{2}=\gamma\right)$ from (8) and the distribution of $\gamma$, we get

$$
\begin{aligned}
P_{\text {out }}^{c}(R, P)= & \int_{\gamma=0}^{\infty} P_{\text {out }}^{c}\left(R, P \mid\|\mathbf{h}\|^{2}=\gamma\right) p_{\gamma}(\gamma) d \gamma \\
\leq & \int_{\gamma=\gamma_{0}}^{\gamma_{1}} N\left(1-\frac{\gamma_{0}}{\gamma}\right)^{(t-1)} p_{\gamma}(\gamma) d \gamma \\
& +\int_{\gamma=\gamma_{1}}^{\infty} 1 p_{\gamma}(\gamma) d \gamma
\end{aligned}
$$




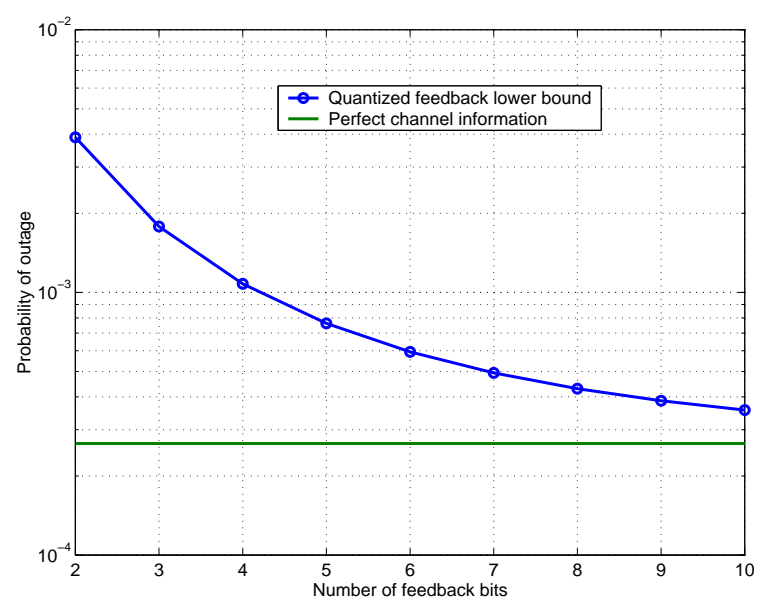

Figure 1: Improvement in outage probability with increasing number of feedback bits for four transmit antennas with a single receive antenna at $\mathrm{SNR}=10 \mathrm{~dB}$, as predicted by Theorem 1 .

The second term in (17) above can be simplified as

$$
\int_{\gamma=\gamma_{1}}^{\infty} 1 p_{\gamma}(\gamma) d \gamma=1-F_{\gamma}\left(\gamma_{1}\right)
$$

where $F_{\gamma}(\gamma)$ is cumulative distribution function of $\gamma$ given by

$$
F_{\gamma}(\gamma)=1-e^{-\gamma} \sum_{k=0}^{t-1} \frac{\gamma^{k}}{k !} .
$$

The first term in (17) can be simplified as follows. We have

$$
\begin{aligned}
\int_{\gamma=\gamma_{0}}^{\gamma_{1}} N\left(1-\frac{\gamma_{0}}{\gamma}\right)^{(t-1)} p_{\gamma}(\gamma) d \gamma= \\
\quad \int_{\gamma=\gamma_{0}}^{\gamma_{1}} \frac{N}{(t-1) !}\left(1-\frac{\gamma_{0}}{\gamma}\right)^{(t-1)} \gamma^{t-1} e^{-\gamma} d \gamma \\
=\frac{N}{(t-1) !} \int_{\gamma=\gamma_{0}}^{\gamma_{1}}\left(\gamma-\gamma_{0}\right)^{t-1} e^{-\gamma} d \gamma \\
=\frac{N}{(t-1) !} \int_{u=0}^{\gamma_{1}-\gamma_{0}} u^{t-1} e^{-u} e^{-\gamma_{0}} d u \\
=N e^{-\gamma_{0}} F_{\gamma}\left(\gamma_{1}-\gamma_{0}\right),
\end{aligned}
$$

where we have used the substitution $u=\gamma-\gamma_{0}$ in (20). Using (18) and (21) in (17) and using $P_{\text {out }}(R, P)=$ $1-P_{\text {out }}^{c}(R, P)$, we get

$$
\begin{aligned}
& P_{\text {out }}(R, P) \geq \\
& \quad F_{\gamma}\left(\gamma_{1}\right)-N e^{-\gamma_{0}} F_{\gamma}\left(\gamma_{1}-\gamma_{0}\right)
\end{aligned}
$$

$$
\begin{aligned}
= & \left(1-e^{-\gamma_{1}} \sum_{k=0}^{t-1} \frac{\gamma_{1}^{k}}{k !}\right) \\
& -N e^{-\gamma_{0}}\left(1-e^{-\left(\gamma_{1}-\gamma_{0}\right)} \sum_{k=0}^{t-1} \frac{\left(\gamma_{1}-\gamma_{0}\right)^{k}}{k !}\right) \\
= & 1-N e^{-\gamma_{0}}+e^{-\gamma_{1}} \sum_{k=0}^{t-1}\left(\frac{N\left(\gamma_{1}-\gamma_{0}\right)^{k}-\gamma_{1}^{k}}{k !}\right)
\end{aligned}
$$

\section{References}

[1] E. Telatar, "Capacity of multi-antenna gaussian channels," ATEST-Bell Labs Internal Tech. Memo, June 1995.

[2] V. Tarokh, N. Seshadri, and A. Calderbank, "Spacetime codes for high data rate wireless communication:performance criterion and code construction," IEEE Trans. on Info. Theory, vol. 44, pp. 744-765, March 1998.

[3] A. Narula, M.D. Trott, and G.W. Wornell, "Performance limits of coded diversity methods for transmitter antenna arrays," IEEE Transactions on Information Theory, pp. 2418-2433, November 1999.

[4] S. Bhashyam, A. Sabharwal, and B. Aazhang, "Feedback gain in multiple antenna systems," IEEE trans. on communications, vol. 50, pp. 785-798, May 2002.

[5] A. Sabharwal, E. Erkip, and B. Aazhang, "On channel state information in multiple antenna block fading channels," in ISITA, Hawaii, 2000.

[6] K. K. Mukkavilli, A. Sabharwal, and B. Aazhang, "Design of multiple antenna coding schemes with channel feedback," in Thirty fifth Asilomar Conference on Signals, Systems and Computers, 2001, pp. 1009-1013.

[7] R.W. Heath Jr. and A. Paulraj, "A simple scheme for transmit diversity using partial channel feedback," in Thirty Second Asilomar Conference on Signals, Systems and Computers, 1998, pp. 1073-1078.

[8] A. Narula, M.J. Lopez, M.D. Trott, and G.W. Wornell, "Efficient use of side information in multiple-antenna data transmission over fading channels," IEEE Journal on selected areas in communications, pp. 1423-1436, October 1998.

[9] E. Visotsky and U. Madhow, "Space-time transmit precoding with imperfect feedback," IEEE transactions on Information Theory, vol. 47, no. 6, pp. 2632-2639, September 2001.

[10] S. A. Jafar and A. Goldsmith, "On optimality of beamforming for multiple antenna systems," in ISIT, 2001.

[11] K. K. Mukkavilli, A. Sabharwal, E. Erkip and B.Aazhang, "On Beamforming with Finite Rate Feedback for Multiple Antenna Systems," submitted to IEEE trans. on Information Theory, 2002.

[12] L.H. Ozarow, S. Shamai, and A.D. Wyner, "Information theoretic considerations for cellular mobile radio," IEEE transactions on Vehicular Technology, vol. 43, pp. 359378, May 1994. 